\title{
LA ACREDITACIÓN EN CONTEXTO DE CAMBIO: EL CASO DE LAS CARRERAS DE INGENIERÍA EN LA ARGENTINA ${ }^{1}$
}

\author{
ERnesto ViLlanUeVA*
}

\author{
Recebido em: 11 de julho de 2008 Aprovado em: 11 de setembro de 2008
}

\begin{abstract}
*Licenciado en Sociología, por la Universidad de Buenos Aires. Es especialista en temas de política universitaria y evaluación y acreditación de la calidad en educación superior. Actualmente es miembro de la Comisión Nacional de Evaluación y Acreditación Universitaria de Argentina (CONEAU) - organismo del cual ha sido presidente en varios períodos - y profesor titular en la Universidad Nacional de Quilmes.
\end{abstract}

Resumen: Afirmar la importancia clave que tiene la educación superior en el desarrollo de cualquier sociedad es un lugar común en las discusiones políticas y académicas sobre la materia. No obstante, dicha afirmación no resuelve la pregunta sobre qué tipo de educación, para qué desarrollo, para qué sociedad. Esto implica reconocer el carácter histórico y político de esa relación: ya que la respuesta a la pregunta sobre qué educación para qué sociedad no ha sido ni puede ser siempre igual y porque cualquiera sea la respuesta, la misma siempre deberá inscribirse en un proyecto político que piense los problemas de la sociedad en su conjunto.

Con estos presupuestos como horizonte, proponemos una reflexión sobre la situación y las perspectivas de la educación superior en América Latina: se trata de una mirada que esboza un balance sobre los cambios ocurridos en los últimos años, para considerar, a continuación, el actual contexto de la educación superior de la región y los desafíos frente a los cuales deberán generarse nuevas respuestas. Pretendemos sumar un aporte al debate que pone en el centro la pregunta sobre qué educación podemos y debemos promover para construir una sociedad con desarrollo productivo y cultural autónomos, con diálogo y participación en el escenario internacional, con equidad, integración y solidaridad.

Palabras clave: Educación superior. Universidad. América Latina.

\section{ACCREDITATION IN THE CONTEXT OF CHANGE: THE CASE OF THE ENGINEERING PROGRAMS IN ARGENTINA}

Abstract: It is common place within academic and political discussions on higher education to affirm its key role in the development of any society. However, this assertion does not solve the question about what kind of education, to what development, for what society. This implies the recognition of the historical and political character of that relationship, since the answer to the question on what education for what society has not been nor can it always be the same; and whatever the answer, it always has to be part of a political project that views the problems of the society as a whole. With those assumptions on the horizon, we propose a reflection on the situation and perspectives of higher education in Latin America: it is a view that, first, assesses the changes occurred during the last years, to then consider the present context of higher education in the region and the challenges which must generate new answers in order to be faced. We attempt to make a contribution to the debate that poses in the center the question of what education we can and must promote in order to build a society with an autonomous cultural and productive development; with dialog and participation in the international scenario; and also with equity, integration and solidarity.

Key-words: Higher education. University. Latin America.

1 Una exposición oral de este escrito se hizo en el workshop organizado por INQAHEE (Internacional Network for Quality Assurance Agencies in Higher Education, en Buenos Aires el día 13 de mayo de 2008. 


\section{Introducción}

A partir de 1995, la educación superior argentina pasó a estar regulada por una nueva $l^{2} y^{2}$. Entre las novedades de la nueva legislación, se establecía la creación de una agencia de evaluación y acreditación de instituciones y carreras universitarias (la Comisión Nacional de Evaluación y Acreditación Universitaria, CONEAU) y la puesta en marcha de los correspondientes procesos de evaluación y acreditación. Esta última sería de carácter obligatoria para las universidades privadas, para todas las carreras de posgrado y para aquellas carreras de grado cuyo ejercicio profesional pusiera en riesgo de modo directo la vida, la salud, la seguridad, los derechos, los bienes o la formación de los habitantes (artículo $43^{\circ}$ de la ley). En principio, este recorte recayó sobre las carreras de Medicina, Ingeniería, Veterinaria y Agronomía.

La primera convocatoria para acreditación se hizo para las carreras de Medicina; en una etapa siguiente se trabajó con las carreras de Ingeniería, lo cual implicó una serie de cambios y desafíos tanto para la agencia, como para sus técnicos, sus herramientas de trabajo y, también, para las comunidades académicas involucradas.

Este trabajo busca transmitir la experiencia de la CONEAU en la acreditación de Ingeniería. Para ello, se describe la situación anterior a la acreditación; se presentan los desafíos a los que se enfrentó la agencia y se analiza el proceso de acreditación, haciendo hincapié en los elementos decisivos que la gestión de dicho proceso tuvo para el mejoramiento de la calidad de las carreras en cuestión. Dado el volumen de carreras analizadas, la acreditación se organizó por regiones consideradas sucesivamente, lo que permitió ajustar los procedimientos e instrumentos a lo largo del proceso. La relevancia de los planes de mejora se hizo evidente sobre la marcha y derivó en el desarrollo de una metodología para la elaboración de esos planes en la fase de autoevaluación. Este trabajo se detiene en describir esta innovación metodológica.

Por último, se intenta dar cuenta de un ciclo de cambio en el cual la comunidad universitaria, la Agencia y el gobierno -orientando la inversión estataldesempeñaron cada una un rol fundamental para el mejoramiento de la calidad de las carreras de Ingeniería.

\section{La Situación de las Universidades Previo a la Acreditación}

En 1988 fue creado el CONFEDI: Consejo Federal de Decanos de Ingeniería de la República Argentina. Esta instancia llevó adelante un proceso de reflexión

2 Ley de educación superior $n^{\circ} 24.521$ 
y de elaboración de estrategias para el mejoramiento de las carreras de Ingeniería que terminó convirtiéndose en el impulso inicial hacia la acreditación.

El CONFEDI elaboró dos documentos muy importantes como antecedentes del proceso de acreditación: Libro Azul (1996): "Unificación Curricular en la Enseñanza de las Ingenierías en la República Argentina"; Libro Verde (2000): "Propuesta de Acreditación de Carreras de Grado de Ingeniería en la República Argentina".

Ambos textos constituyeron los insumos fundamentales para la elaboración de los estándares y criterios para la acreditación de las carreras de Ingeniería que fueron aprobados por el Ministerio de Educación en consulta con el Consejo de Universidades.

A la vez, esos textos posibilitaron un proceso de unificación curricular de las carreras en 21 especialidades, lo cual puso a todas las unidades académicas en condiciones similares desde el punto de vista curricular, reduciendo la gran heterogeneidad de programas que existían anteriormente.

En diciembre de 2001 se aprobaron las normas específicas de calidad para el primer grupo de 13 especialidades de Ingeniería sujetas a acreditación. A la vista está la primera complejidad con la cual tuvo que lidiar la CONEAU para organizar el proceso de acreditación. Normalmente, las facultades ofrecen varias de estas especialidades o carreras de Ingeniería. La organización académica departamental no es la predominante en la tradición argentina y es conocida la tendencia a la autonomización de los espacios académicos. Las facultades exhibían una variedad de fórmulas de articulación y convivencia entre las carreras de Ingeniería de manera tal que resultaba muy difícil aislar cada carrera como unidad de análisis en las evaluaciones.

Por otro lado, el campo académico y profesional de la Ingeniería se encontraba en una situación de marcado deterioro, producto de un contexto económico marcado por la desindustrialización, la recesión económica, la crisis del Estado y la desocupación de profesionales. Estos indicadores y la tasa de desempleo entre los ingenieros desalentaban a los jóvenes para elegir esa profesión. Considerado desde el punto de vista de las carreras, se llegaba a la realidad extrema de carreras vacías de alumnos que mantenían sus plantas docentes y su capacidad instalada, lo que en definitiva mostraba comunidades académicas con fuertes trabas y escasa agilidad para el cambio. La inercia en la dinámica interna se combinaba con un contexto adverso.

No obstante esta situación, a partir de las propuestas del CONFEDI la comunidad universitaria de Ingeniería llevó a cabo un debate y se fue consensuando un modelo de Ingeniero, que se expresó en criterios y estándares de calidad por 
encima del nivel medio real a fin de que operaran como un modelo a alcanzar. Asimismo, se les dio a las instituciones un año de plazo para su adaptación. Dicho modelo dio impulso al cambio: se exigió práctica profesional supervisada, investigación, infraestructura, vinculación con el medio a carreras que habían sufrido un fuerte deterioro.

\section{El Inicio de la Acreditación de Carreras de Ingeniería: Entre la Experiencia y Nuevos los Desafíos}

De acuerdo con los estándares establecidos, se distinguieron 17 tipos de ingenierías para acreditar. Antes que se cumpliera el año de plazo para la adaptación de las carreras a los estándares de calidad, la Agencia convocó a una acreditación voluntaria en el entendimiento de que serviría para afinar los instrumentos. Esta iniciativa generó una situación novedosa y plagada de desafíos para la Agencia: se presentaron 186 carreras, lo que significaban un $77,5 \%$ del total, muchas más que las que esperaba la Agencia.

Por un lado, el elevado número de carreras que se presentaron permite conjeturar que las universidades percibieron que la acreditación sería un factor posibilitador de los cambios en el diseño curricular que se estaban impulsando. En este sentido, se puede sostener que la acreditación fue percibida como punto de apoyo para los sectores internos proclives al cambio, en alianza tácita con la Agencia.

Por otro lado, al iniciarse el proceso de acreditación de las carreras de grado en Ingeniería se contaba con la experiencia de lo realizado en Medicina; sin embargo, frente a las 24 carreras de Medicina con las que había trabajado la Agencia en la respectiva acreditación, ahora pasaba a tener que organizar procesos para 240 carreras, que son el total de las carreras de ingeniería en condiciones de presentarse. Este salto de escala tuvo consecuencias diversas. La presencia de la agencia de acreditación en el sistema universitario se multiplicó y adquirió mayor visibilidad, dado que sus decisiones afectaban a mayor cantidad de actores.

Al mismo tiempo, esta mayor escala implicó desafíos técnicos nuevos, ya que el universo de programas a acreditar presentó una heterogeneidad mayor, con más dificultades de ser contenidos en los instrumentos de evaluación estandarizados.

La cuestión de los estándares presentó también una serie de dificultades para la Agencia. En la Argentina, las normas de calidad para la acreditación (denominadas estándares) son establecidas por el Ministerio de Educación en 
consulta con el Consejo de Universidades, conformado por rectores de universidades estatales y privadas; la CONEAU las aplica mediante la organización de procesos de evaluación, pero no las define.

Como se dijo, las normas específicas para la acreditación de las Ingenierías se habían aprobado en diciembre del 2001, días antes de que una gran crisis económica e institucional pusiera en jaque al país entero. Esas normas había sido resultado de un prolongado debate en el ámbito de la comunidad universitaria de Ingeniería que permitió la generación de un consenso en torno a la necesidad de la acreditación para mejorar la calidad de las carreras existentes. Más aún, previo a la acreditación, diversas facultades de Ingeniería ya habían emprendido reformas para el mejoramiento. Sin embargo, esas normas de calidad establecidas estaban por encima del nivel medio que cumplían las carreras, con lo cual la CONEAU se enfrentó con el desafío de cómo conducir política y técnicamente un proceso de acreditación aplicando normas de calidad que se suponen un piso mínimo pero que en la realidad son objetivamente muy difíciles de cumplir por parte de la mayoría de las carreras existentes, aun teniendo en cuenta que la comunidad académica venía mostrando una disposición al cambio.

\section{La Puesta en Marcha de la Acreditación: Entre el Compromiso con el Mejoramiento de la Calidad y la Distancia con los Pisos Mínimos de Calidad Fijados en los Estándares}

El proceso de acreditación de las Ingenierías tuvo 3 claves que posibilitaron un análisis en consonancia con el proceso de cambio en las carreras, a saber: 1) facultades como unidad de análisis; 2) acreditación con compromisos; 3) evaluación de planes de mejora.

1 - Facultades como unidad de análisis. La unidad de análisis de la acreditación es en principio la carrera; es el sujeto al que se le imputa la decisión de acreditación. En el proceso de acreditación de Medicina no surgieron mayores dificultades en torno a este asunto, dado que las carreras de Medicina son en general la principal o la única actividad de las facultades de Medicina, dando lugar prácticamente a una asimilación entre el nivel facultad y el nivel carrera, al menos funcional para implementar un diagnóstico. En cambio, las facultades de Ingeniería normalmente ofrecen un conjunto de especialidades, a veces, con diferencias importantes entre ellas en cuanto a calidad. Por otra parte, normalmente las especialidades se encuentran articuladas a nivel curricular. 
En las evaluaciones se procuró diferenciar la capacidad de educar de la unidad académica de la situación de las diferentes carreras, intentando que el diagnóstico sobre la unidad académica volcado en la resolución de acreditación -que tiene como sujeto cada carrera- fuera uniforme en todas las carreras dictadas en dicha unidad académica. Al mismo tiempo, se procuraron diferenciar los requerimientos y compromisos para la acreditación según si el resorte para la solución de los problemas estuviera en la unidad académica o en la carrera.

2 - Acreditación con compromisos. La CONEAU contaba con la figura de acreditación por 3 años que había creado en el marco de la acreditación de Medicina, mediante la Ordenanza 005/99 que establecía los procedimientos y pautas para la acreditación de carreras de grado. En ella se fijaba, entre otras importantes cuestiones, una auto evaluación guiada a través de instrumentos técnicos proporcionados por la CONEAU (con una duración estipulada en alrededor de 4 meses) y las alternativas en poder de la Comisión para resolver sobre la acreditación de una carrera:

a) acreditar por 6 años para las carreras que cumplan con el perfil de acreditación previsto en los estándares;

b) acreditar por 3 años en los casos en los que, no obstante no haberse logrado el perfil previsto, hubiese elementos suficientes para considerar que la carrera desarrolla efectivamente estrategias de mejoramiento cuyo impacto debiera lograr el perfil antedicho;

c) no acreditar.

En un documento institucional de 2002, en el cual se analiza el impacto de su labor, la Comisión daba cuenta de acreditar por 3 años como resultado posible de la evaluación: "En la gestión del proceso de acreditación de medicina, la CONEAU debió desarrollar fórmulas operativas mediadoras entre el estado real de las carreras en el sistema y el «deber ser» alojado en los estándares" (CONEAU, 2002, p. 43) En esta dirección, se resolvió diferenciar la validez de la acreditación en 3 y 6 años. Además, en el enfoque de la acreditación se remarcó el mejoramiento de la calidad como el objetivo central del proceso $\mathrm{y}$, en consecuencia, se jerarquizaron los planes de mejoramiento surgidos de la autoevaluación; cuando se juzgó que éstos no eran adecuados para cumplir con los estándares, los comités de pares establecieron requerimientos para la acreditación a partir de los cuales las instituciones tuvieron la oportunidad de generar nuevos planes de mejoramiento, los cuales a la hora de acreditar se 
transformaron en compromisos. Se puede sostener que los procedimientos diseñados por la Comisión buscaron evitar una penalización con duras repercusiones para las instituciones que no alcanzaren los estándares o en una cristalización de situaciones de desigualdad en el seno del sistema universitario (debe tenerse en cuenta que sobre la calidad repercuten una variedad de factores, entre otros, los recursos al alcance de las instituciones y la asignación presupuestaria en su interior). Se privilegió construir un escenario de cambios encaminados al mejoramiento de la calidad, pactados en sus contenidos y plazos con la Comisión, que promete elevar el nivel de la formación de los médicos. La política de la CONEAU en ese sentido fue mantener el rigor de las evaluaciones (sin flexibilizar las exigencias de los estándares) ofreciendo un camino de mejoramiento para las instituciones cuyas carreras no cumplían perfectamente los estándares. Al mismo tiempo, dicha política permitió minimizar los conflictos que podrían haberse suscitado entre la CONEAU y las instituciones en la primera experiencia de acreditación de carreras de grado." ${ }^{3}$

3 - Evaluación de planes de mejora. Los compromisos se sostienen en la evaluación de planes de mejora. En la experiencia de acreditación de Medicina se abrió un espacio para la presentación de planes de mejora nuevos a partir de los requerimientos que los pares establecían para la acreditación en la primera instancia de evaluación. Antes de la resolución de la CONEAU, las instituciones toman conocimiento del dictamen del Comité de pares evaluadores. Si la carrera no alcanza los estándares de calidad, los pares incluyen requerimientos para la acreditación en dicho dictamen. Las instituciones tienen un tiempo para responder a la vista del dictamen y la respuesta es evaluada por los pares.

Durante la acreditación de Medicina se advirtió la relevancia los planes de mejora y se brindaron pautas mínimas para la presentación de los planes. Sin embargo, fue en la acreditación de Ingeniería cuando estos planes tomaron más importancia para la toma de decisión en torno a la acreditación. De ahí la necesidad de intervenir para lograr el diseño de mejores planes, lo cual a su vez llevó al desarrollo de una metodología para la elaboración de planes de mejora en la fase de autoevaluación, la cual se expone a continuación.

3 Se podría haber optado por no acreditar a las carreras que no cumplieran con los estándares y recomendar suspender la inscripción de nuevos alumnos hasta tanto se subsanen las deficiencias encontradas, tal como establece el artículo 76 de la LES. Hay que señalar que el Decreto 499/95 establece que la acreditación es condición necesaria para el reconocimiento oficial y la validez nacional del título, con lo cual el alcance de la no acreditación sería mayor. Desacreditar no es frecuente en la experiencia internacional; por ejemplo, en EEUU existe la acreditación a plazos y sólo se penaliza a los francamente malos. 


\section{Planeamiento de Acciones de Desarrollo Institucional}

En base a elementos de sociología de las organizaciones, de administración y políticas públicas y del planeamiento institucional se desarrolló una metodología para la elaboración de planes de mejora de las carreras de ingeniería. Se trata de una herramienta para que las acciones a implementar para solucionar los déficits de las carreras con respecto a los estándares de calidad se sostengan en un diagnóstico integrado de problemas. En otras palabras, una metodología que desalienta diagnósticos fragmentados por dimensión evaluada o por estándar. Por ello, ellos quedaron agrupados en cinco dimensiones: contexto institucional, docencia, investigación, infraestructura y equipamiento, recursos humanos. Se intenta que los planes de mejora funcionen como palancas para el cambio, sea para cumplir con los requisitos mínimos para la acreditación o también para alcanzar estándares de excelencia por encima de los mínimos requeridos. A su vez, estos planes de mejora configuran la cuarta etapa de la autoevaluación, a cargo de la propia institución universitaria. Consideremos, entonces, las etapas planificadas:

\section{Etapa 1: Análisis de la situación actual de la carrera e identifica-} ción de los posibles déficits. La elaboración de un diagnóstico de la situación actual de la carrera implica un esfuerzo de análisis global que implica un recorrido por todas las dimensiones. Es una excelente oportunidad para detectar potencialidades o recursos inexplorados y ver en qué medida son relevantes para superar posibles déficits. Se entienden como déficits los obstáculos o brechas que impiden que se cumpla con los criterios de calidad requeridos para el dictado de la carrera.

Etapa 2: Definición de la naturaleza de los problemas. No es el objeto del informe de auto evaluación presentar una descripción pormenorizada de los déficits sino avanzar en la detección de los problemas que les dan origen. Por ello, una vez definidos los déficits, y, en primera instancia, dentro de cada dimensión, se buscará la naturaleza del problema que podría provocarlos.

Se presentan 6 categorías de problemas teniendo presente que cada déficit podría estar vinculado con más de una categoría. 


\section{Naturaleza de los problemas}

1) Normativa, procedimientos o diseño curricular: inexistencia, superposición o contradicción en ordenanzas, resoluciones o normas; falta de adecuación en los reglamentos de concursos o en los reglamentos de correlatividades; inconvenientes en las condiciones de inscripción y reinscripción de alumnos; incorrecta designación del personal, falta o desactualización de convenios, resoluciones de aprobación de planes de estudio.

2) Coordinación institucional, planificación y seguimiento: superposición de competencias, falta de coordinación entre autoridades o instancias de decisión, ausencia de planificación y programación de las actividades de dirección, carencia de comisiones específicas, incorrecta articulación del circuito administrativo, falta de definición de lineamientos de investigación, falta de claridad en los objetivos a corto y mediano plazo para el desarrollo de tares cotidianas, falta de difusión de los reglamentos, estatutos o normativas, ausencia de seguimiento y control del currículo; de las actividades de investigación, desarrollo, extensión y transferencia así como también relacionados con el desempeño de los alumnos.

3) Organización interna: debilidades en el funcionamiento de departamentos, cátedras, oficinas de registro de alumnos, etc.; ineficiencia en el circuito administrativo referido a legajos de personal, emisión de títulos, actas de exámenes, confidencialidad y resguardo de la información; debilidades en la conexión entre direcciones y personal, servicios de

información internos y externos; incorrecto manejo de la documentación; escasa accesibilidad a la biblioteca y otras instalaciones; inadecuada distribución de funciones para la docencia, investigación, seguimiento de alumnos o tutorías.

4) Desarrollo del currículo: inconvenientes en la formación inicial de los alumnos o en la formación docente-pedagógica, inadecuadas metodologías para la evaluación, incorrecto grado de actualización de los conocimientos del plan de estudios, falta de contenidos en el plan de estudios.

5) Recursos humanos: incorrecta asignación de personal según jerarquía o área; magnitud inapropiada de la planta docente y no docente; inconvenientes en la calificación de los recursos docentes y no docentes disponibles; distorsión en la asignación de la planta según las capacidades requeridas para la tarea; falta de personal crítico para llevar a cabo tareas especializadas; falta de continuidad de la planta, falta de capacidad para el desarrollo de actividades de investigación y de docencia (en función de la formación y dedicación).

6) Recursos físicos y presupuesto: falta de adecuación de las instalaciones, incorrecta distribución de la infraestructura y el equipamiento, inconvenientes en el origen o asignación de los recursos financieros (inadecuada distribución para proyectos de investigación, mantenimiento, pago a docentes, construcciones, equipamiento informático, biblioteca, becas, etc.). 


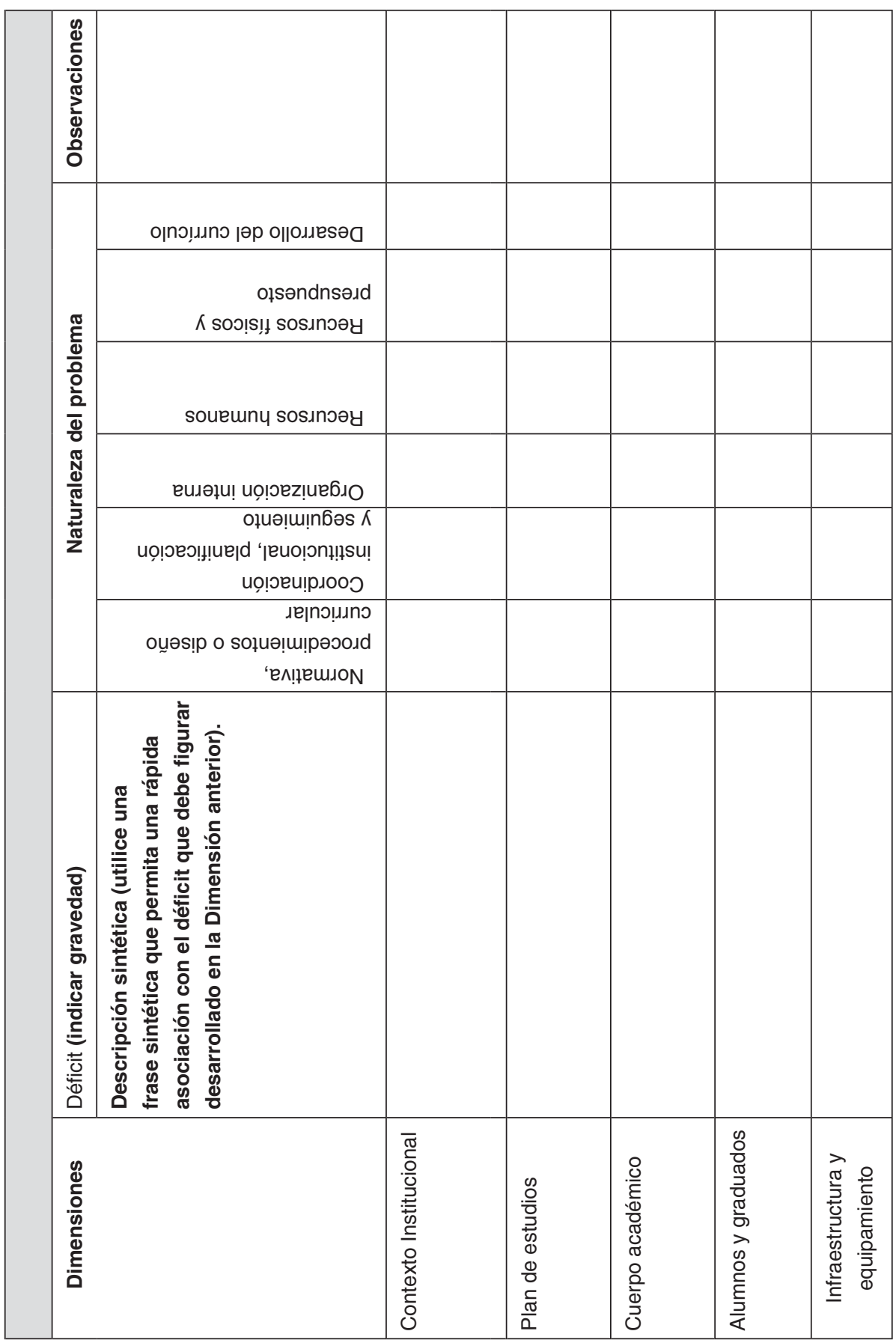


Tomemos un ejemplo: una carrera con un desgranamiento importante en las etapas iniciales, un déficit originado en una cantidad insuficiente de docentes o una formación deficiente de los estudiantes o un plan de estudios excesivo en relación con la cantidad de horas de clase. A la vez, supongamos que esa carrera muestra pocas actividades de investigación y transferencia, resultado de la falta de personal calificado para llevar adelante este tipo de tareas así como también al escaso tiempo disponible por el cuerpo académico (a raíz de una excesiva carga docente) para adquirir la formación necesaria o desarrollar este tipo de trabajos. En esta situación hipotética, surge la necesidad de armar planes de mejoramiento para modificar el plan de estudios y para modificar la composición del cuerpo académico. Ello implica una decisión de planificación a nivel institucional acerca de las metas a lograr en aspectos vinculados con investigación y transferencia.

Para sistematizar el trabajo de análisis de la calidad de la carrera, de detección de déficits y definición de problemas se proporciona un modelo de matriz o planilla a ser usada como elemento de trabajo (ver próximo gráfico). El análisis y descripción de la información que contiene constituye la síntesis que se solicita al final de cada dimensión.

Etapa 3: Construcción de la agenda integrada. La construcción de la agenda requiere de un análisis integrado de los déficits que presenta la carrera para el cumplimiento de los estándares de calidad que se han identificado en cada una las dimensiones (contexto institucional, plan de estudios y formación, etc.), la naturaleza de los problemas (de normativa, de coordinación institucional, etc.) con la que fueron asociados y su gravedad. Este análisis tiene como finalidad sistematizar los problemas que, por relacionarse con el mismo déficit, por abarcar varios de ellos, o por similitud en la naturaleza del problema, puedan enfrentarse con acciones vinculadas.

La agenda que se defina permitirá establecer los objetivos del plan de mejoramiento, es decir, los cambios que fueran necesarios para superar las dificultades encontradas.

Etapa 4: Elaboración del plan de mejoramiento. El plan de mejoramiento es el documento que expone la relación entre objetivos, responsables de realizar las actividades y recursos que la carrera necesita para alcanzar el cumplimiento de los criterios de calidad que establece la resolución ministerial. Incluye también la descripción 
de las actividades, la previsión de los recursos necesarios (según las fuentes a las que la carrera tiene acceso y otras que deberá procurar), la programación de las tareas a implementar y toda otra información o documentación que contribuya a evaluar la pertinencia, razonabilidad y viabilidad del plan.

A diferencia de los objetivos, referidos al qué se desea lograr, las actividades establecen en cómo alcanzar los logros propuestos (las tareas o el camino a seguir). Para alcanzar el logro de los objetivos, las actividades pueden agruparse en distintas categorías como por ejemplo:

a) elaboración de normas, diseños curriculares o procedimientos;

b) obtención de recursos físicos o financieros;

c) obtención y asignación de recursos humanos;

d) capacitación del personal;

e) decisiones a adoptar por diferentes actores involucrados.

El plan de mejoramiento debe contener un apartado por cada categoría de actividades a desarrollar para el cumplimiento de los objetivos. A los efectos de poder evaluar la pertinencia, razonabilidad y viabilidad del plan se debe incluir: actores involucrados en la toma de decisiones o participantes en la realización de las tareas, convenios y acuerdos firmados o a firmar, planos, convocatorias a concursos, contenido de los planes o programas de capacitación, evaluaciones, encuestas a implementar, listado de bibliografía o equipamiento a adquirir, licitaciones, entre otros.

\section{Orientación de la Inversión Estatal para el Mejoramiento de la Calidad}

Al término del proceso de acreditación de las carreras de Ingeniería, la Agencia redactó un diagnóstico de la situación general de las carreras de ingeniería. Dicho informe operó como un puente para transferir los diagnósticos sobre calidad construidos mediante evaluación por pares hacia la esfera de las políticas públicas para el sector universitario.

Esta práctica que se inició con Ingeniería se convirtió en rutina cada vez se culmina el proceso de acreditación de una titulación, constituyendo una manera de aprovechar el panorama razonablemente completo de las carreras 
existentes en el país al que se accede a través de la acreditación. ${ }^{4}$ Utilizando esa información, el Ministerio de Educación ha creado programas de promoción de la calidad de carreras de ingeniería y también de agronomía, que incluyen financiamiento especial en relación a las necesidades detectadas.

\section{Conclusiones: un Ciclo de Cambio}

Aunque la innovación metodológica para la elaboración de planes de mejora fue bien recibida por las instituciones, lo cierto es que se siguen registrando diferencias en los estilos de presentación de las autoevaluaciones. Esto es, la metodología sugerida por la Agencia fue implementada de manera desigual por parte de las universidades. En algunos casos se observan mejoras significativas. La metodología indica un camino para analizar las causas de los problemas de calidad que reclama un alto compromiso en el interior de las universidades.

En términos generales, el peso puesto en la elaboración de planes de mejora y en su posterior evaluación, brindó una base más sólida para establecer los compromisos que debían asumirse. Ello se verifica en el nuevo proceso de acreditación que se está desarrollando en el que se comprueba un alto grado de cumplimiento de los compromisos. En este sentido, puede afirmarse que se está cerrando un ciclo de cambio de las carreras de Ingeniería que no obtuvieron la acreditación por 6 años, en el cual se constatan mejoras en la calidad.

En definitiva, la experiencia que se ha analizado en este trabajo encontró un modo de resolver en los hechos la tensión entre los objetivos de aseguramiento y mejoramiento de la calidad que afecta a todo sistema de evaluación y acreditación de la educación superior.

\section{Referencia}

CONEAU. Contribuciones para un análisis del impacto del sistema de evaluación y acreditación. Buenos Aires, 2002.

4 Véase: CONEAU-SPU "Panorama General de las Carreras de Ingeniería de la Argentina", febrero de 2005; CONEAU-MECyT "Situación de las Carreras de Ingeniería Agronómica de la Argentina", noviembre de 2005 y el reciente informe de CONEAU-MECyT, "Situación actual de las Carreras de Bioquímica y Farmacia de la Argentina, julio de 2007. 